\title{
FLORESCIMENTO DE TANGERINEIRAS 'PONKAN' (Citrus reticulata Blanco) SUBMETIDAS A DIFERENTES PERÍODOS DE TEMPERATURA INVERNAL ${ }^{1}$
}

\author{
FLÁVIA MARA VIEIRA LELIS ${ }^{2}$, DALMO LOPES DE SIQUEIRA ${ }^{3}$, DIERLEI DOS SANTOS ${ }^{4}$
}

RESUMO - O trabalho foi desenvolvido com o objetivo de avaliar o efeito do acúmulo de temperaturas abaixo de $20^{\circ} \mathrm{C}$, sobre a floração de tangerineiras 'Ponkan' (Citrus reticulata Blanco). Foi adotado o delineamento inteiramente casualizado, com seis tratamentos e quatro repetições. Os tratamentos foram formados por sete datas de transferência das mudas, mantidas a céu aberto, para a câmara de crescimento. Foram utilizadas plantas de tangerineira 'Ponkan' enxertadas sobre limoeiro 'Cravo', cultivadas em recipientes, que inicialmente foram mantidas a céu aberto. O experimento foi iniciado no dia 16-05-2007 (início do período de redução da temperatura na região) e conduzido até 17-08-2005. Quinzenalmente, quatro plantas que estavam a céu aberto foram transferidas para câmara de crescimento, com temperaturas de $27^{\circ} \mathrm{C} / 20^{\circ} \mathrm{C}$ (dia/noite), umidade relativa de $75 \%$, fotoperíodo de 12 horas e radiação fotossinteticamente ativa de $170 \mu \mathrm{mol} \mathrm{m} \mathrm{m}^{-2} \mathrm{~s}^{-1}$. Foram avaliados a data do florescimento e o número total de flores de todas as plantas. Quanto maior foi o tempo de permanência das plantas a céu aberto em temperaturas inferiores a $20^{\circ} \mathrm{C}$, maiores foram a antecipação do florescimento e o número de flores produzidas.

Termos para indexação: Floração dos citros, temperatura, ambiente controlado.

\section{FLOWERING OF 'PONKAN' MANDARIN (Citrus reticulata Blanco) SUBMITTED TO DIFFERENTS PERIODS OF WINTER TEMPERATURE}

\begin{abstract}
This work aimed to evaluate the effect of low temperature accumulation below $20^{\circ} \mathrm{C}$, on the flowering of mandarin (Citrus reticulata Blanco cv. 'Ponkan'). A completely randomized design with six treatments and four replications was used in this experiment. The treatments comprised seven different dates of transference of 'Ponkan' plants growing in open field into growth room. The 'Ponkan' trees used in this experiment were originated from grafting onto Rangpur lime, grown in containers. The experiment began on 05-16-2007, when the cold season initiated. In 15-days intervals, four plants were removed randomly from the open field and taken into the growth room, with controlled temperature and humidity. The date of the flowering and total number of flowers were evaluated. The longest time of maintenance of the plants in the open field in temperatures below $20^{\circ} \mathrm{C}$ advanced the flowering and increased the number of produced flowers.
\end{abstract}

Index terms: citrus flowering, temperature, controlled environment.

O conhecimento sobre a floração dos citros ainda é escasso em virtude das dificuldades técnicas para o seu estudo. Como exemplo, pode ser citada a impossibilidade de se separar morfologicamente, ou de acordo com sua posição na planta, os meristemas reprodutivos dos vegetativos, antes da brotação da primavera, visto que nem todas as gemas do ramo produzem flores (Lord \& Eckard, 1985). Entretanto, o destino da gema (vegetativa ou reprodutiva) não é definido ao acaso, mas depende de condições fisiológicas e de desenvolvimento de cada ramo e de condições do ambiente (Reuther, 1973).

As modificações morfológicas nos meristemas das plantas cítricas podem ser notadas somente após o repouso do inverno (Guardiola, 1981; Iwahori \& Oohata, 1981; Lord \& Eckard,
1985). Temperaturas que oscilam entre 13 a $15^{\circ} \mathrm{C}$ durante o dia e $10 \mathrm{a} 13^{\circ} \mathrm{C}$ à noite são consideradas promotoras da floração, destacando-se por sua eficiência no estímulo do processo (Moss, 1969).

Apesar da existência de pesquisas procurando determinar as faixas ideais para o início da floração dos citros, não se sabe ao certo quais são os limites máximos para a indução do processo, acreditando-se estar em torno de $19^{\circ} \mathrm{C}$, sendo que temperaturas superiores a $22^{\circ} \mathrm{C}$ já são ineficientes (Davenport, 1990).

Para Krajewski \& Rabe (1995), o efeito das baixas temperaturas sobre a indução floral dos citros não está demonstrado de forma definitiva, pois seu efeito sobre o aumento no número de flores é seguido também por aumentos

\footnotetext{
${ }^{1}$ (Trabalho 210-07). Recebido em: 04-09-2007. Aceito para publicação em: 15-05-2008.

${ }^{2}$ Aluna de graduação do curso de Agronomia da Universidade Federal de Viçosa, Departamento de Fitotecnia, Viçosa- MG, 36570-000, e-mail: fvieiralelis@yahoo.com.br.

${ }^{3}$ Eng. Agr., DS, Professor Associado I da Universidade Federal de Viçosa , Departamento de Fitotecnia, Viçosa- MG, 36570-000, e-mail: siqueira@ ufv.br. ${ }^{4}$ Aluno de graduação do curso de Agronomia da Universidade Federal de Viçosa, Departamento de Fitotecnia, Viçosa- MG, 36570-000, Bolsista do CNPq, e-mail: dierlei@ pop.com.br.
} 
significativos nos números de brotações vegetativas. $\mathrm{N}$ a $\mathrm{s}$ experiências de Garcia-Luis et al. (1992), com gemas de tangerineira 'Satsuma' (C. unshiu Marcov.) cultivadas in vitro, foi verificado que temperaturas baixas exercem função dupla sobre as gemas, que consiste em quebra de dormência e indução do florescimento. Também se constatou dormência mais profunda nas gemas floríferas quando comparadas às vegetativas.

Após determinado tempo expostas às condições de baixas temperaturas, torna-se necessário o contato com temperaturas mais elevadas, a fim de se completar seu florescimento. Os primeiros sinais da morfogênese são detectados somente ao se iniciarem as brotações de primavera (Guardiola, 1981; Iwahori \& Oohata, 1981; Lord \& Eckard, 1985).

O florescimento satisfatório ocorre quando há acúmulo de 750 a 1.000 horas de temperaturas abaixo de $20^{\circ} \mathrm{C}$ sem interrupções no período por temperaturas que estimulam a emissão de novas brotações, ou seja, sem a ocorrência de temperaturas máximas acima de $26,6^{\circ} \mathrm{C}$ e mínimas superiores a $21,1^{\circ} \mathrm{C}$ por mais de sete dias seguidos (Albrigo et al., 2002).

Com base no acúmulo de baixas temperaturas, Albrigo et al. (2002) estabeleceram três diferentes níveis de indução de floração. Consideraram o nível de indução como baixo quando ocorre menos de 750 horas abaixo de $20^{\circ} \mathrm{C}$. Nessa condição, o estímulo não é suficiente para induzir uma floração significativa, que possa resultar em produção econômica. O nível médio de indução ocorre quando o número de horas abaixo de $20^{\circ} \mathrm{C}$ varia entre 750 e 1.200 horas. Nesse caso, pode ocorrer indução suficiente para garantir uma produção econômica. Por último, propuseram o nível alto, que ocorre quando o número de horas de acúmulo de temperaturas abaixo de $20^{\circ} \mathrm{C}$ está acima de 1.200 horas, sendo o estímulo suficiente para induzir a uma boa produção.

Outro fator importante na indução de floração é o déficit hídrico, principalmente nas regiões de clima tropical, onde as temperaturas não são suficientemente baixas para promover a indução. De forma similar às baixas temperaturas, a relação entre a intensidade e a duração do déficit hídrico e a intensidade de floração é aparentemente quantitativa (Southwick \& Davenport 1986).

A Zona da Mata Mineira está dentro dos limites do clima tropical mesotérmico brando úmido, com predomínio de temperaturas amenas durante todo o ano (média anual de $18^{\circ} \mathrm{C}$ a $19^{\circ} \mathrm{C}$ ) e ocorrência de duas estações climáticas anuais (chuvosa e seca). O inverno tem, pelo menos, um mês de temperatura média inferior a $15^{\circ} \mathrm{C}$, mas nunca abaixo de $10^{\circ} \mathrm{C}$, sendo junho e julho os meses mais frios. O verão é ameno, e a média do mês mais quente é inferior a $22^{\circ} \mathrm{C}$ (Nimer, 1977). A precipitação média é de aproximadamente $1.315 \mathrm{~mm}$ anuais (Melo \& Antunes 1979). Como as flores dos citros, nessa região, são emitidas após um período onde ocorrem, simultaneamente, temperaturas baixas e estresse hídrico, não foram determinados a importância e o efeito de cada um dos fatores (temperatura e estresse hídrico) sobre a floração dos citros.

O objetivo do trabalho foi quantificar a necessidade de horas de frio para a indução do florescimento e determinar se o frio, isoladamente, provoca indução floral em tangerineiras
Ponkan (Citrus reticulata Blanco) nas condições climáticas do município de Viçosa-MG.

O experimento foi conduzido em câmara de crescimento do setor de Fruticultura localizado no Câmpus da Universidade Federal de Viçosa, situado a $21^{\circ} 05^{\prime}$ de latitude sul e $42^{\circ} 51^{\prime}$ de longitude oeste, com $650 \mathrm{~m}$ de altitude. O clima da região é do tipo Cwa, segundo classificação de Köppen.

Foram utilizadas plantas, mantidas a céu aberto (temperatura ambiente), de tangerineira 'Ponkan' (Citrus reticulata Blanco) enxertada sobre limoeiro 'Cravo' (Citrus limonia Osbeck), com dois anos de idade. O diâmetro médio do caule a $5 \mathrm{~cm}$ acima do ponto de enxertia foi de $20,4 \mathrm{~mm}$ e o comprimento médio das plantas foi de 1,24 m. Essas plantas foram cultivadas em substrato comercial e mantidas em recipientes com $24 \mathrm{~cm}$ de altura e $26 \mathrm{~cm}$ de diâmetro.

O trabalho foi conduzido com o delineamento inteiramente casualizado, com 6 tratamentos e 4 repetições, com uma planta por parcela. A partir do dia 16-05-2005 até o dia 17-08-2005, quatro plantas foram retiradas periodicamente do local onde estavam mantidas a céu aberto e levadas para a câmara de crescimento, totalizando seis datas de transferência, que foram consideradas como tratamentos.

A câmara foi regulada com temperaturas adequadas ao crescimento vegetativo $\left(27^{\circ} \mathrm{C} / 20^{\circ} \mathrm{C}\right.$ dia/noite $)$ e, portanto, inadequadas à indução da floração. A umidade relativa estava em 75\%,o fotoperíodo usado foi de 12 horas, e a Radiação Fotossinteticamente Ativa (RAF), de $170 \mu \mathrm{mol} \mathrm{m}^{-2} \mathrm{~s}^{-1}$.

Dessa forma, quanto mais tempo as plantas permaneceram a céu aberto, maior foi o tempo em que estiveram expostas às baixas temperaturas do inverno. A temperatura e a umidade relativa foram registradas por um datalogger (Sato), posicionado no local onde as plantas foram mantidas a céu aberto, em intervalos de 15 minutos.

Todas as plantas (plantas a céu aberto e plantas no interior da câmara) foram irrigadas diariamente para evitar que $o$ estresse hídrico fosse um fator de interferência e foram observadas diariamente para avaliação do florescimento. Para cada planta, foi anotada a data do florescimento, que foi usada para cálculo do número de dias compreendidos entre a transferência das plantas para a câmara (temperaturas nãoindutoras) e o início do florescimento. Também foi avaliado, para cada planta, o número total de flores produzidas.

$\mathrm{O}$ acúmulo de horas de frio, que foi representado pelo número de horas com temperaturas abaixo de $20^{\circ} \mathrm{C}$, para cada tratamento, foi avaliado a partir do dia 25 de abril até a data de transferência para a câmara. Somente a partir desta data é que não mais ocorreram temperaturas máximas acima de $26,6^{\circ} \mathrm{C}$ e mínimas superiores a $21,1^{\circ} \mathrm{C}$ por mais de sete dias seguidos.

As variáveis avaliadas foram submetidas à análise de variância (ANOVA) e, posteriormente, à análise de regressão, utilizando o software livre R (R DEVELOPMENT CORE TEAM, 2005).

Durante todo o tempo em que as plantas permaneceram expostas à temperatura ambiente, mesmo após o acúmulo das horas de frio necessárias para a quebra da dormência e a emissão de flores, que, segundo Albrigo et al. (2002), deve ser acima de 
800 a 1.000 horas de temperaturas abaixo de $20^{\circ} \mathrm{C}$, elas não emitiram nenhuma brotação, seja reprodutiva, seja vegetativa. Tal fato ocorreu devido à ausência de temperaturas elevadas (acima de $20^{\circ} \mathrm{C}$ ), que são necessárias para o estímulo da brotação, confirmando as observações de Guardiola (1981); Iwahori \& Oohata (1981); Lord \& Eckard (1985).

Por outro lado, as plantas que não foram expostas às baixas temperaturas e que, portanto, acumularam poucas horas de frio (transferências efetuadas em 16-05-2005 e 30-05-2005), praticamente, não produziram flores, mesmo expostas por longos períodos à temperatura favorável à brotação (Tabela 1).

$\mathrm{O}$ acúmulo de horas de frio apresentou efeito quadrático sobre o tempo necessário para a emissão de brotos reprodutivos (Figura 1). Plantas expostas pouco tempo às baixas temperaturas, como as transferidas para a câmara de crescimento no dia 16-052005, acumularam apenas 354,5 horas de frio e levaram, em média, 65,6 dias para emitirem flores. Em contrapartida, plantas expostas a 1.637,5 horas de frio, que correspondem ao ponto de mínimo na curva da Figura 1, floresceram em apenas 3,4 dias após serem transferidas para a câmara de crescimento.

O acúmulo de horas de frio também apresentou efeito quadrático sobre o número médio de flores emitidas por planta (Figura 2), sendo que plantas expostas pouco tempo às baixas temperaturas praticamente não produziram flores, mesmo permanecendo um período longo no interior da câmara de crescimento, em condições favoráveis ao seu desenvolvimento. O número máximo de flores produzidas por planta, que foi, em média, 146 flores, ocorreu quando elas acumularam 1.904,0 horas de frio, correspondendo ao ponto de máximo, na curva.

Com base no acúmulo de baixas temperaturas necessárias para a floração dos citros (Tabela 1 e Figura 2), é possível estabelecer três diferentes níveis de indução de floração da tangerineira 'Ponkan', de forma semelhante à realizada por Albrigo et al. (2002).

Nesse caso, os valores obtidos foram aproximados aos dos referidos autores, podendo ser adotada a mesma classificação, ou seja, o nível de indução é baixo quando ocorre acúmulo de menos de 750 horas de temperaturas abaixo de $20^{\circ} \mathrm{C}$. Nessa condição, o estímulo não é suficiente para induzir uma floração significativa. $O$ nível médio de indução ocorre quando o número de horas abaixo de $20^{\circ} \mathrm{C}$ varia entre 750 e 1.200 horas, e o nível alto ocorre quando o número de horas de acúmulo de temperaturas abaixo de $20^{\circ} \mathrm{C}$ está acima de 1.200 horas, sendo o estímulo suficiente para induzir a uma boa produção.

Para as condições climáticas de Viçosa, o estresse hídrico não foi necessário para a floração da tangerineira 'Ponkan, tendo em vista que todas as plantas usadas no experimento foram irrigadas diariamente.
TABELA 1- Efeito do acúmulo de horas de frio em tangerineiras 'Ponkan' sobre o número de dias para o início do florescimento e sobre o número médio de flores por planta, em função das datas de transferência para a câmara de crescimento.

\begin{tabular}{|c|c|c|c|}
\hline $\begin{array}{c}\text { Data da } \\
\text { Transferência }\end{array}$ & $\begin{array}{l}\text { Horas de Frio } \\
\text { Acumuladas } \\
\text { (temperatura } \\
<20^{\circ} \mathrm{C} \text { ) }\end{array}$ & $\begin{array}{l}\text { Número de dias } \\
\text { para a floração }\end{array}$ & $\begin{array}{l}\text { Número de } \\
\text { flores } \\
\text { produzidas }\end{array}$ \\
\hline $16-05-2005$ & 354,5 & 65,6 & 7,4 \\
\hline $30-05-2005$ & 617,0 & 26,0 & 28,0 \\
\hline $16-06-2005$ & 887,5 & 18,3 & 90,4 \\
\hline 01-07-2007 & $1.161,5$ & 11,0 & 133,7 \\
\hline $15-07-2005$ & $1.418,5$ & 10,0 & 109,7 \\
\hline 01-08-2005 & $1.733,0$ & 5,8 & 134,7 \\
\hline $17-08-2005$ & $1.994,0$ & 0,5 & 145,0 \\
\hline & \multicolumn{3}{|c|}{ Análise de Variância } \\
\hline & $\mathrm{P}$-valor $\operatorname{Pr}(>\mathrm{F})$ & $<0,001$ & $<0,001$ \\
\hline & CV $(\%)$ & 21,93 & 20,08 \\
\hline
\end{tabular}

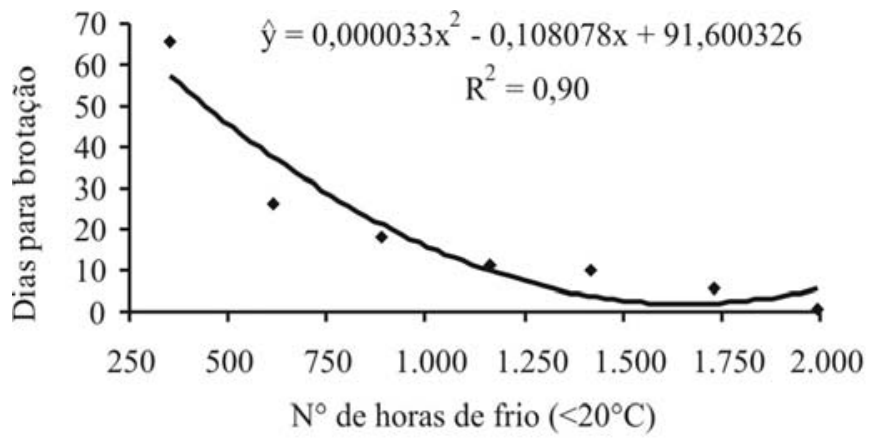

FIGURA 1- Estimativa do efeito do número de horas de frio acumuladas - HFA (menores que $20^{\circ} \mathrm{C}$ ) sobre o início da floração de tangerineiras 'Ponkan'.

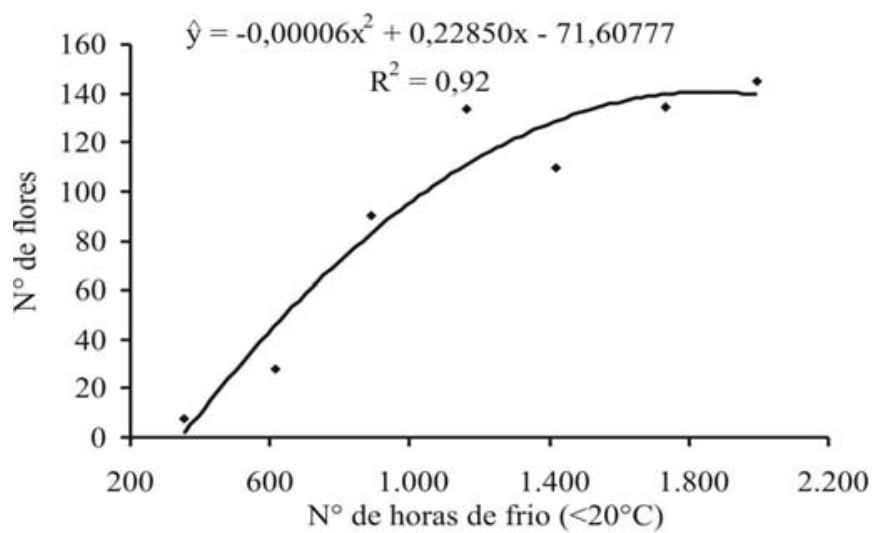

FIGURA 2- Estimativa do efeito do número de horas de frio acumuladas - HFA (menores que $20^{\circ} \mathrm{C}$ ) sobre o número médio de flores produzidas por tangerineiras 'Ponkan'. 


\section{REFERÊNCIAS}

ALBRIGO, L.G.; VALIENTE, J.I.; BECK, H.W Flowering expert system development for a phenology based citrus decision support system. Acta Horticulturae, Wageningen, v. 584, p.247254,2002

DAVENPORT, T.L. Citrus flowering. Horticultural Reviews, New York, v.12, p.349-408, 1990.

GARCÍA-LUIS, A.; KANDUSER, M.; SANTAMARINA, P.; GUARDIOLA, J. L. Low temperature influence on flowering in Citrus. The separation of inductive and bud dormancy releasing effects. Physiologia Plantarum, Copenhagen, v.86, p.648-652, 1992.

GUARDIOLA, J.L. Flower initiation and development in citrus. Proceedings of the International Society of Citriculture,Washington, v.2, p.242-246, 1981.

IWAHORI, S.; OOHATA, J.T. Control of flowering of Satsuma mandarins (Citrus unshiu Marc.) with gibberellin. Proceedings of the International Society of Citriculture, Washington, v.1, p.247-249, 1981.

KRAJEWSKI, A.J.; RABE, E. Citrus flowering: a critical evaluation. Journal of Horticultural Science, Ashford Kent, v.70, p.357-374, 1995.
LORD, E.M.; ECKARD, M.J. Shoot development in Citrus sinensis L. Osbeck (Washington navel orange): I. Floral and inflorescence ontogeny. Botanical Gazette, Chicago, v.146, p. 320-326, 1985

MELO,M.J.S.; ANTUNES,F.Z. Zoneamento climático de Minas Gerais visando à exclusão do cancro-cítrico. Informe Agropecuário, Belo Horizonte, v.5, n.51, p.43-53, 1979

MOSS, G.I. Influence of temperature and photoperiod on flower induction and inflorescence development in sweet orange (Citrus sinensis L. Osbeck). Journal of Horticultural Science, Ashford, v.44, p.311-320, 1969 .

NIMER, E. Clima. In: GOLDENBERG, C. (Ed.). Geografia do Brasil: região sudeste. Rio de Janeiro: IBGE, 1938. p.51-89.

RDEVELOPMENTCORETEAM. R: A language and environment for statistical computing. Viana: R Foundation for Statistical Computing, 2005. Disponível em: <http://www.R-project.org>.

REUTHER, W. Climate and citrus behavior. In: REUTHER, W. (Ed.). The citrus industry. Berkeley: University of California, 1973. v.3, p.280-337.

SOUTHWICK, S. M.; DAVENPORT, T. L. Characterization of water stress and low temperature effects on flower induction in citrus. Plant Physiology, Bethesda, v.81, p.26-9, 1986. 